\title{
A statistics-respecting bijection between permutation matrices and descending plane partitions without special parts
}

\author{
Markus Fulmek* \\ Faculty of Mathematics \\ University of Vienna \\ Vienna, Austria \\ Markus.Fulmek@univie.ac .at
}

Submitted: Oct 24, 2018; Accepted: Jan 23, 2020; Published: Feb 7, 2020

(c) The author. Released under the CC BY license (International 4.0).

\begin{abstract}
We present a bijection between permutation matrices and descending plane partitions without special parts, which respects two of the statistics considered by Mills, Robbins and Rumsey, and an additional statistic considered by Behrend, Di Francesco and Zinn-Justin.
\end{abstract}

Mathematics Subject Classifications: 05A05, 05A19

\section{Introduction}

It is a well-known fact $[11,5,4]$ that the enumeration

- of descending plane partitions with parts not exceeding $n$ (let us denote the set of these objects by $\mathcal{D}_{n}$ )

- and of alternating sign matrices of dimension $n$ (let us denote the set of these objects by $\left.\mathcal{A}_{n}\right)$

gives the same number:

$$
\left|\mathcal{A}_{n}\right|=\left|\mathcal{D}_{n}\right|=\prod_{j=0}^{n-1} \frac{(3 j+1) !}{(n+j) !} .
$$

*Research supported by the National Research Network "Analytic Combinatorics and Probabilistic Number Theory", funded by the Austrian Science Foundation. 


\subsection{Search for a bijection}

It appears to be quite difficult to find some "natural" bijection

$$
\Phi: \mathcal{A}_{n} \rightarrow \mathcal{D}_{n}
$$

However, there are two additional informations which might help in the search for such bijection:

- There is a quadruplet $(p, i, s, q)$ of statistics for both $\mathcal{A}_{n}$ and $\mathcal{D}_{n}$, i.e. there are functions

$$
\sigma_{A}: \mathcal{A}_{n} \rightarrow \mathbb{Z}^{4} \text { and } \sigma_{D}: \mathcal{D}_{n} \rightarrow \mathbb{Z}^{4}
$$

such that for all $y:=(p, i, s, q) \in \mathbb{Z}^{4}$ the preimages are equinumerous, i.e.,

$$
\left|\sigma_{A}^{-1}(y)\right|=\left|\sigma_{D}^{-1}(y)\right|
$$

(see [3, Theorem 1], the details are given in the next section).

- There are certain subsets of $\mathcal{A}_{n}$ and of $\mathcal{D}_{n}$, namely

- alternating sign matrices with statistic $s=0$ (let us denote this set by $\mathcal{A}_{n}^{0}$; it is, in fact, the set of $n \times n$ permutation matrices),

- descending plane partitions with statistic $s=0$ (let us denote this set by $\mathcal{D}_{n}^{0}$ ),

which are much simpler to understand and for which it is, in fact, quite easy to give "natural" bijections (see below).

So an obvious approach would be to search for a bijection $\Phi$ which respects this quadruplet of statistics $(p, i, s, q)$; in the sense that for all $A \in \mathcal{A}_{n}$ there should hold:

$$
\sigma_{A}(A)=\sigma_{D}(\Phi(A)) \text { for all } A \in \mathcal{A}_{n}
$$

Clearly, such bijection $\Phi$ restricted to the subset $\mathcal{A}_{n}^{0} \subset \mathcal{A}_{n}$ would give a bijection

$$
\Psi: \mathcal{A}_{n}^{0} \rightarrow \mathcal{D}_{n}^{0}
$$

So if we find such "restricted" bijection $\Psi$ which respects the triplet $(p, i, q)$ of statistics in the sense of (2), then we might hope to "extend" it somehow to the desired "full" bijection $\Phi$.

The purpose of this note is to present a simple bijection $\Psi$ which indeed respects the triplet $(p, i, q)$ of statistics in the sense of (2): The construction of $\Psi$ relies on the representation of descending plane partitions as families of non-intersecting lattice paths and on a certain "visualization" of the statistic $i$ (as number of certain entries) for matrices). 


\subsection{Other bijections}

It should be noted that there are other bijections $\mathcal{A}_{n}^{0} \rightarrow \mathcal{D}_{n}^{0}$ : Maybe the simplest one was already mentioned by Lalonde [8, p.981], who referred to the inversion table of a permutation: This table (also called inversion word) gives a (well-known) unique encoding of permutations. The missing link from inversion words to $\mathcal{D}_{n}^{0}$ was explained by Striker [10, Lemma 5]. Striker uses monotone triangles as intermediate objects to establish the bijection between inversion words and $\mathcal{A}_{n}^{0}$, but this intermediate step is not necessary: Instead, we can employ directly the well-known encoding of permutations by inversion words. (We shall call these two mappings $\mathcal{A}_{n}^{0} \rightarrow \mathcal{D}_{n}^{0}$ Striker's and Lalonde's bijection, respectively). Unfortunately, none of these two simple bijections respects the statistic $q$ (see Figure 4). Ayyer [1] presented another (inductively constructed) bijection, which does not respect the statistic $i$ (see [1, p. 1786]).

\subsection{Organization of this note}

This note is organized as follows:

- Section 2 contains basic definitions and background information,

- Section 3 presents a "visualization" of inversions in alternating sign matrices used for our bijection,

- Section 4 presents a representation of descending plane partitions as families of nonintersecting lattice paths (in the "obvious" way: for the expert it will suffice to look at Figure 2),

- Section 5 presents a bijection $\Psi: \mathcal{A}_{n}^{0} \rightarrow \mathcal{D}_{n}^{0}$ which respects the triplet $(p, i, q)$ of statistics.

\section{Background information}

For reader's convenience, we recall some background information.

\subsection{Descending plane partitions}

Here is the definition of descending plane partitions as given by Mills, Robbins and Rumsey $[9$, Definitions 2-4]:

Definition 1 (descending plane partition). A descending plane partition is an array $\pi=$ $\left(a_{i, j}\right), 1 \leqslant i \leqslant j<\infty$, of positive integers

$$
\begin{aligned}
& \pi=\begin{array}{ccccccc}
a_{1,1} & a_{1,2} & a_{1,3} & \ldots & & \ldots & a_{1, \mu_{1}} \\
& a_{2,2} & a_{2,3} & \ldots & \ldots & a_{2, \mu_{2}} &
\end{array} \\
& \begin{array}{ccc} 
& \cdots & \\
a_{k, k} & \cdots & a_{k, \mu_{k}}
\end{array}
\end{aligned}
$$


such that

1. rows are weakly decreasing, i.e., $a_{i, j} \geqslant a_{i, j+1}$ for all $i=1, \ldots, k$ and $i \leqslant j<\mu_{i}$,

2. columns are strictly decreasing, i.e., $a_{i, j}>a_{i+1, j}$ for all $i=1, \ldots, k-1$ and $i<j \leqslant$ $\mu_{i+1}$,

3. $a_{i, i}>\mu_{i}-i+1$ for all $i=1, \ldots, k$,

4. $a_{i, i} \leqslant \mu_{i-1}-i+2$ for all $i=2, \ldots, k$.

Clearly, conditions 3 and 4 imply

$$
\mu_{1} \geqslant \mu_{2} \geqslant \cdots \geqslant \mu_{k} \geqslant k \text {. }
$$

The parts of a descending plane partition are the numbers (with repetitions) that appear in the array. The empty array, which we denote by $\varnothing$, is explicitly allowed.

A descending plane partition $\pi$ where no part is greater than $n$ (i.e., $\pi$ has at most $n-1$ rows) is said to have dimension $n$. (So a descending plane partition of dimension $n$ may also be viewed as a descending plane partition of dimension $k$, for all $k>n$.)

By the length of row $i$ in descending plane partition $\pi$ we define the number of parts it contains (i.e., $\mu_{i}-i+1$ ). So we may rephrase conditions 3 and 4 as follows:

$3^{\prime}$. The first part of row $i$ is greater than the length of row $i$ for $i=1, \ldots, k$,

$4^{\prime}$. The first part of row $i$ is less or equal than the length of the preceding row $i-1$ for $i=2, \ldots, k$.

A part $a_{i, j}$ in a descending plane partition is called special if it does not exceed the number of parts to its left (in its row $i$ ), i.e., if

$$
a_{i, j} \leqslant j-i .
$$

Example 2. A typical example is the array

$$
\begin{array}{lllll}
6 & 6 & 6 & 4 & \underline{2} \\
& 5 & 3 & \underline{2} & \underline{1} \\
& & 2 & &
\end{array}
$$

with 3 rows and 10 parts (written in descending order)

$$
6,6,6,5,4,3,2, \underline{2}, \underline{2}, \underline{1}
$$

three of which are special parts (indicated as underlined numbers; note that the 2 in the last row is not a special part):

$$
\underline{2}, \underline{2}, \underline{1} \text {. }
$$

(This is the example $D_{0}$ considered by Lalonde [7, Fig. 1].)

From now on, we shall use the shorthand $D P P$ for descending plane partitions. 


\section{$2.2 \quad$ Alternating sign matrices}

Here is the definition of alternating sign matrices as given by Mills, Robbins and Rumsey, see [9, Definition 1]:

Definition 3 (alternating sign matrix). An alternating sign matrix of dimension $n$ is an $n \times n$ square matrix which satisfies

- all entries are $1,-1$ or 0 ,

- every row and column has sum 1 ,

- in every row and column the nonzero entries alternate in sign.

Suppose that $A=\left(A_{i, j}\right)_{1}^{n}$ is an alternating sign matrix of dimension $n$. Then the number of inversions in $A$ is defined to be $[9$, p. 344]

$$
\sum_{\substack{1 \leqslant i<k \leqslant n \\ 1 \leqslant l<j \leqslant n}} A_{i, j} A_{k, l} .
$$

Example 4. The following matrix is an example of an alternating sign matrix of dimension 5:

$$
\left[\begin{array}{ccccc}
0 & 1 & 0 & 0 & 0 \\
0 & 0 & 1 & 0 & 0 \\
1 & -1 & 0 & 1 & 0 \\
0 & 1 & 0 & -1 & 1 \\
0 & 0 & 0 & 1 & 0
\end{array}\right]
$$

From now on, we shall use the shorthand $A S M$ for alternating sign matrices.

\subsection{The Mills-Robbins-Rumsey conjecture}

Here is the Conjecture of Mills, Robbins and Rumsey [9, Conjecture 3], slightly rephrased to fit our exposition:

Theorem 5. Suppose that $n, p, i, s$ are nonnegative integers, $0 \leqslant p \leqslant n-1$. Let $\mathcal{A}_{n}(p, i, s)$ be the set of ASMs such that

1. the size of the matrix is $n \times n$ (i.e., its dimension is $n$ ),

2. the number of 0 's to the left of the 1 in the first row is $p$,

3. the number of -1 's in the matrix is $s$,

4. the number of inversions in the matrix is $i+s$.

On the other hand, let $\mathcal{D}_{n}(p, i, s)$ be the set of DPPs such that

1. no part exceeds $n$ (i.e., the dimension of the DPP is $n$ ), 
2. there are exactly $p$ parts equal to $n$,

3. there are exactly s special parts,

4. there are a total of $i+s$ parts.

Then $\mathcal{A}_{n}(p, i, s)$ and $\mathcal{D}_{n}(p, i, s)$ have the same cardinality - the sets $\mathcal{A}_{n}$ and $\mathcal{D}_{n}$ are equidistributed with respect to the triplet of statistics $(p, i, s)$. $1]$.

This conjecture was proved by Behrend, Di Francesco and Zinn-Justin [2, Theorem

\subsection{The fourth statistic given by Behrend, Di Francesco and Zinn-Justin}

In [3], Behrend, Di Francesco and Zinn-Justin presented a fourth statistic $q$ for matrices and DPPs and showed that matrices and DPPs are equidistributed with respect to the quadruplet of statistics $(p, i, s, q)[3$, Theorem 1]: This statistic $q$ is

- for $n$-dimensional ASMs equal to the number of 0 's to the right of the 1 in the last row,

- for $n$-dimensional DPPs equal to the number of parts $n-1$ plus the number of rows of length $n-1$.

\subsection{Permutation matrices and inversions}

Let $\sigma \in \mathfrak{S}_{n}$ be a permutation of the first $n$ natural numbers $\{1,2, \ldots, n\}$.

\subsubsection{Inversions of a permutation}

Recall that an inversion of $\sigma$ is a pair $(i, j)$ such that $i<j$ and $\sigma(i)>\sigma(j)$. For the number $\operatorname{inv}(\sigma)$ of all inversions of $\sigma$ we have $0 \leqslant \operatorname{inv}(\sigma) \leqslant \frac{n(n-1)}{2}$.

We may assign to $\sigma$ its inversion word $\left(a_{1}, a_{2}, \ldots, a_{n-1}\right)$, where $a_{k}$ is the number of inversions $(i, j)$ with $\sigma(j)=k, k=1,2, \ldots, n-1$. Clearly we have $0 \leqslant a_{k} \leqslant n-k$ and $a_{1}+a_{2}+\cdots+a_{n-1}=\operatorname{inv}(\sigma)$.

Considering the one-line notation

$$
[\sigma(1), \sigma(2), \ldots, \sigma(n)]
$$

of $\sigma$, the inversion word's $k$-th entry $a_{k}$ is simply the number of elements to the left of $k$ (in the one-line notation) which are greater than $k$, and it is easy to see that every word $\left(b_{1}, b_{2}, \ldots, b_{n-1}\right)$ with $0 \leqslant b_{k} \leqslant n-k$ determines a unique permutation: Inversion words are, in this sense, just another "encoding" for permutations. 


\subsubsection{Permutation matrices}

A permutation $\sigma \in \mathfrak{S}_{n}$ can be represented by an $n \times n$-matrix $M$ with entries

$$
M_{i, j}=\delta_{i, \sigma(j)}
$$

(where $\delta_{x, y}$ denotes Kronecker's delta: $\delta_{x, y}=1$ if $x=y, \delta_{x, y}=0$ if $x \neq y$; this is the transpose of the usual permutation matrix). We call this matrix the permutation matrix of $\sigma$ : Clearly, it contains precisely one entry 1 in every row and column.

Example 6. Let $n=6$ and $\sigma \in \mathfrak{S}_{6}$ be the permutation with one-line notation

$$
\sigma=[6,3,1,4,2,5]
$$

The corresponding permutation matrix is

$$
\left[\begin{array}{llllll}
0 & 0 & 1 & 0 & 0 & 0 \\
0 & 0 & 0 & 0 & 1 & 0 \\
0 & 1 & 0 & 0 & 0 & 0 \\
0 & 0 & 0 & 1 & 0 & 0 \\
0 & 0 & 0 & 0 & 0 & 1 \\
1 & 0 & 0 & 0 & 0 & 0
\end{array}\right]
$$

and the corresponding inversion word is

$$
(2,3,1,1,1)
$$

Note that every permutation matrix is an ASM (which does not contain entries -1), and that the definition of inversions (3) for ASMs is a generalization of the number of inversions of a permutation (see also Section 3.2).

\section{Inversions in ASMs}

\subsection{Orientation of cells in ASMs}

Definition 7. By a cell in an ASM $A$ we simply mean the position $(i, j)$ (at row $i$ and column $j$ ). If $A_{i, j}=0$, we call this a zero-cell, otherwise a non-zero cell: So, non-zero cells can be either 1 -cells or $(-1)$-cells.

Observe that for every zero-cell $(i, j)$ in an ASM there must be

- to the left or to the right (or both) of $(i, j)$ a closest non-zero cell in row $i$, precisely one of which must be a 1-cell: If this 1 -cell lies to the left of $(i, j)$, then call this cell left-oriented, otherwise right-oriented,

- above or below (or both) $(i, j)$ a closest non-zero cell in column $j$, precisely one of which must be a 1-cell: If this 1 -cell lies below $(i, j)$, then call this cell downoriented, otherwise up-oriented. 
A zero-cell $(i, j)$ is called

- $r d-c e l l$, if it is right-oriented and down-oriented.

- lu-cell, if it is left-oriented and up-oriented.

Remark: rd-cells and lu-cells correspond to two of the four vertex states in the six vertex model which are replaced by zeroes by the well-known bijection between square ice and alternating sign matrices (the remaining two of the altogether six possible vertex states are replaced by +1 and -1 , see [6, Figure 5]).

\subsection{Inversions of matrices}

Observe that the $r d$-cells in the permutation matrix $A=\left(\delta_{i, \sigma(j)}\right)$ of some permutation $\sigma \in \mathfrak{S}_{n}$ are in one-to-one correspondence with the inversions of $\sigma:(i, j)$ is a rd-cell if

- entry 1 in row $i$ is in column $y=\sigma^{-1}(i)>j$ (to the right of column $j$ ),

- and entry 1 in column $j$ is in row $\sigma(j)>i$ (below row $i$ ).

This is equivalent to

$$
j<y \text { and } \sigma(j)>(i=\sigma(y)),
$$

i.e., $(j, y)$ is an inversion of the permutation $\sigma$.

More generally, observe that the fourfold sum (3), which defines the number of inversions of an ASM, may be rewritten as follows

$$
\sum_{(i, l)=(1,1)}^{(n, n)} \sum_{(k, j)=(i+1, l+1)}^{(n, n)} A_{i, j} A_{k, l} .
$$

Now observe that the inner sum is simply the product

$$
\left(\sum_{k=i+1}^{n} A_{k, l}\right)\left(\sum_{j=l+1}^{n} A_{i, j}\right) .
$$

A moment's thought shows that this product is equal to

$$
[(i, l) \text { is a }(-1)-\text { cell or an } \mathrm{rd}-\text { cell }] .
$$

Here, we used Iverson's bracket:

$$
\text { [assertion] }:=\left\{\begin{array}{lc}
1 & \text { if the assertion is true } \\
0 & \text { otherwise }
\end{array}\right.
$$

So the number of inversions of an ASM $A$ (according to (3)) is equal to the number of rd-cells of $A$ plus the number of $(-1)$-cells of $A$. 
The left picture shows the two paths constructed for $\mathrm{rd}-$ cell $(1,1)$ : The first cell of crossing of these paths is lu-cell $(4,6)$, so $(1,1)$ is mapped to $(4,6)$.

The right picture shows the two paths constructed for $\mathrm{rd}-$-cell $(3,2)$ : The first cell of crossing of these paths is lu-cell $(6,5)$, so $(3,2)$ is mapped to $(6,5)$. Observe that these paths also meet (but do not cross) at the -1 -cell $(5,4)$.
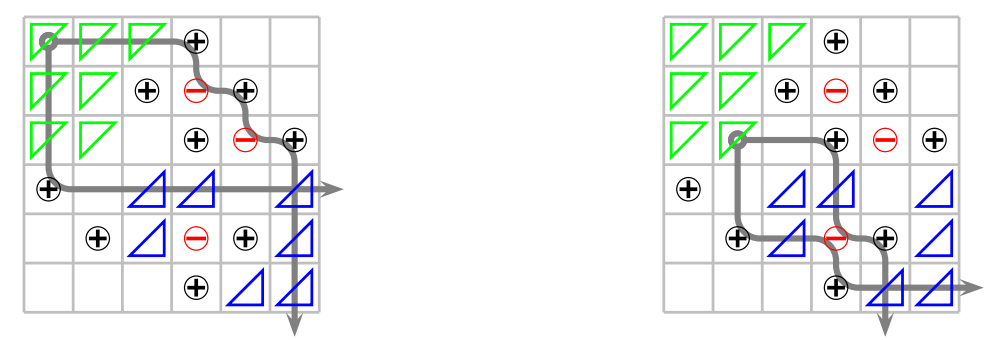

Figure 1: The bijection mapping rd-cells to lu-cells.

\subsection{Quadruplet of statistics, reformulated}

These considerations immediately lead to the following reformulation of the quadruplet of statistics $(p, i, s, q)$ :

\begin{tabular}{|c|l|l|}
\hline \multicolumn{2}{|c|}{ Definition of the statistic $f:$} \\
\hline$f$ & for $D \in \mathcal{D}_{n}:$ & for $A \in \mathcal{A}_{n}:$ \\
\hline$p$ & $\#$ (parts equal to $n)$ & $\#($ rd-cells in first row) \\
$i$ & $\#$ (non-special parts) & $\#($ rd-cells $)$ \\
$s$ & $\#$ (special parts) & $\#((-1)$-entries $)$ \\
$q$ & $\#$ (parts $(n-1))+\#$ (rows of length $(n-1))$ & $\#($ lu-cells in last row) \\
\hline
\end{tabular}

\section{4 rd-cells and lu-cells are equinumerous}

Observe that for every ASM $A$ the number of rd-cells equals the number of lu-cells. We show this by a bijection (see Figure 1): For some rd-cell $(i, j)$, we construct two paths,

- both starting at $(i, j)$,

- both proceeding only horizontally to the right or vertically downwards,

- and both changing horizontal/vertical direction of movement whenever they encounter a non-zero cell.

One of these paths starts horizontally (to the right), the other starts vertically (downwards): The pictures in Figure 1 illustrate this simple idea. Now observe that the paths starting horizontally must necessarily end vertically, and vice versa. Hence the paths must eventually cross at some cell, and any such cell of crossing must necessarily be a lu-cell (the paths could meet but not cross at some $(-1)$-cell, see the right picture in Figure 1): Map $(i, j)$ to the first cell of crossing $(k, l)$ thus obtained. By symmetry (reflection at the second diagonal of $A$ ) it is immediately clear that this construction gives a bijection. 
Consider the following two DPPs of dimension 6:

$$
\begin{array}{llllllllll}
6 & 6 & 6 & 4 & \underline{2} & 6 & 6 & 6 & 5 & 0 \\
& 5 & 3 & \underline{2} & \underline{1} & & 4 & 3 & \underline{1} & \\
& & 2 & & & & & 2 & &
\end{array}
$$

The pictures below show the non-intersecting lattice paths corresponding to the above DPPs:
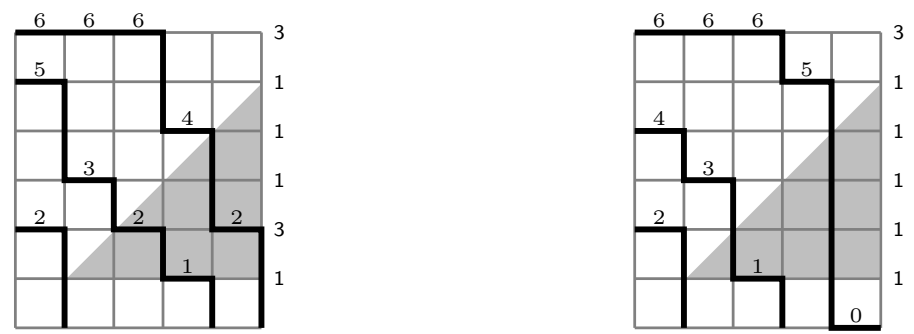

Note that the the right DPP has a zero-padded first row, and that the special parts of these DPPs correspond to the horizontal steps (at heights $>0$ ) in the "special range" below the main diagonal $y=x$ (indicated by the gray triangle).

Figure 2: Representation of DPPs as lattice paths.

\section{Representation of DPPs as lattice paths}

If some row $i$ in a DPP $\pi=\left(a_{i, j}\right)$ is shorter than $a_{i, i}-1$, i.e.,

$$
\delta=\left(a_{i, i}-1\right)-\left(\mu_{i}-i+1\right)>0,
$$

then we pad this row with $\delta$ trailing zeroes (so the length of some row is the number of non-zero parts in that row.)

Now we employ the well-known encoding of (shifted) tableaux as non-intersecting lattice paths; i.e., we encode a DPP $\pi=\left(a_{i, j}\right)$ of dimension $n$ with $r$ rows as an $r$-tuple of non-intersecting lattice paths in the lattice $\mathbb{Z}^{2}$. For reader's convenience, we shall describe the details of this encoding below, but the idea can easily be obtained by looking at the illustrative example in Figure 2.

These lattice paths shall only use horizontal steps to the right or vertical steps downwards, i.e., steps leading from lattice point $(x, y)$ to lattice point $(x+1, y)$ or to lattice point $(x, y-1)$.

The starting points of these $r$ lattice paths are the points

$$
S_{i}:=\left(0, a_{i, i}\right) \text {, }
$$

i.e., the lattice path corresponding to row $i$ starts on the vertical axis at height equal to the first part of row $i$. 
The ending points of these lattice paths are the points

$$
E_{i}:=\left(a_{i, i}-1,0\right) \text {, }
$$

i.e., the lattice path corresponding to row $i$ ends on the horizontal axis and consists of $a_{i, i}-1$ horizontal steps at heights corresponding to the parts of row $i$; including padded zeroes (if any), which correspond to steps at height 0.

It is easy to see that the set $\mathcal{D}_{n}$ of $n$-dimensional DPPs is in bijection with the set of non-intersecting lattice paths as defined above, with no starting point higher than $n$. (But note that in this representation, the number of horizontal steps of a path is equal to the length of the corresponding row plus the number of horizontal steps at height zero.)

\subsection{Bijection between inversion words and DPPs without special parts}

If we have some ("incomplete") lattice path $\mathfrak{p}$ starting in $(0, h)$ and ending in $(x, y)$, where $x \leqslant h-1$ and $y \geqslant 0$, then by the completion of $\mathfrak{p}$ we mean the path obtained by appending to $\mathfrak{p}$

- $y$ vertical steps (downwards)

- and $h-1-x$ horizontal steps (at height 0 , to the right)

(i.e., the completion of $\mathfrak{p}$ ends in $(h-1,0)$ ).

Striker's bijection [10, Lemma 5] between inversion words and DPPs without special parts corresponds to the following construction.

If the inversion word $w=\left(a_{1}, a_{2}, \ldots, a_{n-1}\right)$ equals $(0,0, \ldots, 0)$, then simply assign to $w$ the empty DPP (with no lattice path at all). Otherwise we start with the empty DPP, to which the construction will successively add lattice paths. Let $k$ be the smallest index for which $a_{k}>0$. View $w$ as (the encoding of) a lattice path $\mathfrak{p}$ which starts at $(0, n-k+1)$ and ends at $\left(a_{1}+a_{2}+\cdots+a_{n-1}, 0\right)$, containing $a_{j}$ horizontal steps at height $n-j+1$ for $j=k, k+1, \ldots, n-1$ (see Figure 3). As long as there is an intersection $P=(h, h)$ of $\mathfrak{p}$ with the boundary $y=x$ of the "special region", cut $\mathfrak{p}$ at $P$ in two parts $\mathfrak{p}_{1}$ (containing $\mathfrak{p}$ 's starting point) and $\mathfrak{p}_{2}$ (containing $\mathfrak{p}$ 's ending point), remove any vertical steps at the beginning of $\mathfrak{p}_{2}$, and

- add the completion of $\mathfrak{p}_{1}$ to the (representation of the) DPP under construction;

- if $\mathfrak{p}_{2}$ contains horizontal steps, the first of which starts at some point $Q=\left(h, h^{\prime}\right)$ where $h^{\prime} \leqslant h$, then shift $\mathfrak{p}_{2}$ by $h$ units to the left (so that it starts at $\left(0, h^{\prime}\right)$ ) and set $\mathfrak{p}$ equal to this shifted part; else stop the construction.

If the construction has not yet stopped, then $\mathfrak{p}$ is a lattice path which does not intersect the boundary $y=x$ : Simply add the completion of $\mathfrak{p}$ to the DPP and stop the construction.

See Figure 3 for an illustration of this construction: It is easy to see that it gives always a DPP without special parts and is, in fact, a bijection. 
Consider the inversion word $w=(2,1,3,1,1)$ and "view" it as the encoding $(6,6,5,4,4,4,3,2)$ of the lattice path $\mathfrak{p}$ shown as thick gray line: The construction "cut at the boundary $y=x$, complete and shift" applied to this path gives the three nonintersecting lattice paths shown as thin black lines; these three paths correspond DPP $\begin{array}{lllll}6 & 6 & 5 & 4 & \\ & 4 & 4 & 3 & \text { with three rows }\end{array}$

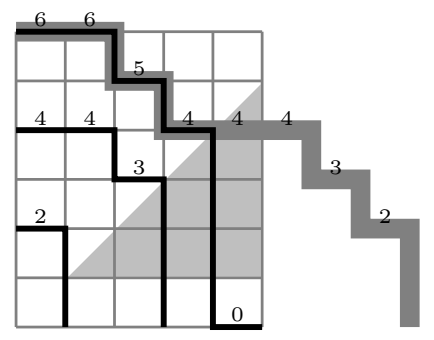

Figure 3: Illustration of Striker's bijection.

\subsection{Striker's and Lalonde's bijection do not respect statistic $q$}

Now it is easy to show by a simple example that neither Lalonde's nor Striker's bijection respect statistic $q$ : Look at Figure 4, where we indicated

- the 1-cells by symbol $\oplus$,

- the (-1)-cells by symbol $\ominus$,

- the rd-cells by small equilateral right-angled triangles, where the sides of the right angle point to the right and downwards,

- and the lu-cells by small equilateral right-angled triangles, where the sides of the right angle point to the left and upwards.

\section{The bijection between DPPs without special parts and per- mutation matrices}

\subsection{The statistic $q$ for DPPs without special parts}

Observe that if a DPP $\pi \in \mathcal{D}_{n}$ without special parts has a row of length $n-1$, this row

- must start with part $n$

- and must not contain parts smaller than $n-1$

(this fact is immediately seen from the lattice path representation of $\pi$ ). 
Consider the 4-dimensional DPP $\pi={ }^{4}{ }_{3}^{4} \frac{4}{2}: q(\pi)=2$, since $\pi$ has one part 3 and one row of length 3 .

The lattice path representation of $\pi$ is shown to the left, the inversion word (needed for both Lalonde's and Striker's bijection) corresponding to $\pi$ is $(3,1,1)$, and the monotone triangle (needed for Striker's "intermediate" bijective step [10]) corresponding to $\pi$ is shown on the top.

Both Striker's and Lalonde's bijection map $\pi$ to the ASM shown to the right, with statistic $q$ equal to 3 (the number of lu-cells, indicated by equilateral right-angled triangles, in the last row is 3). However, a bijection respecting the quadruplet of statistics should map $\pi$ to the ASM shown at the bottom, which is the only ASM of dimension 4 with quadruplet of statistics $(3,5,0,2)$.
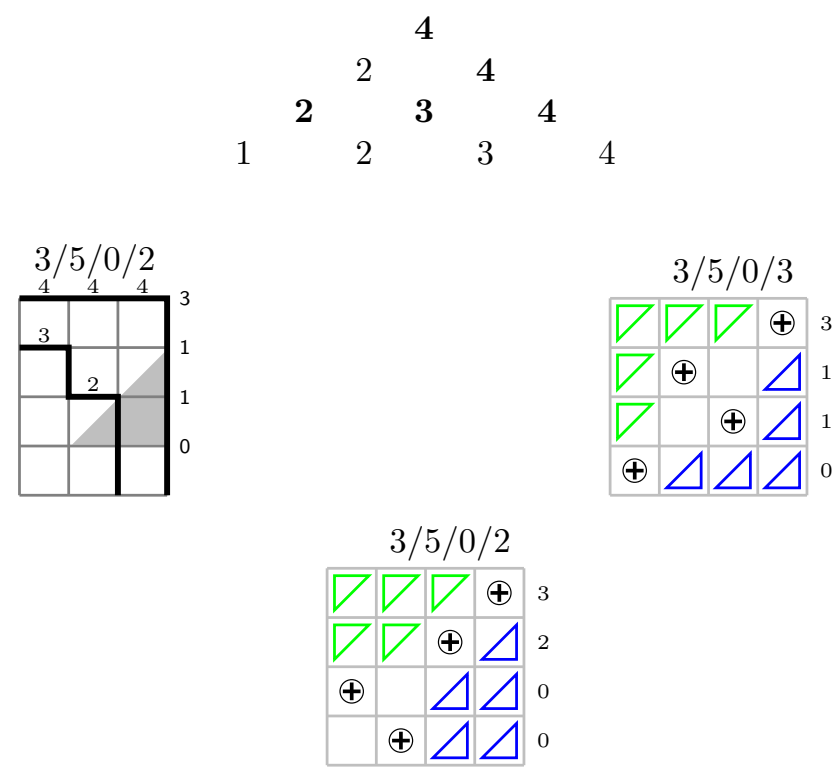

Figure 4: Neither Lalonde's nor Striker's bijection respect statistic $q$. 
Stated otherwise: The somewhat complicated condition "existence of a path of length $n-1 "$ can be simply expressed as

$$
\#(\text { parts equal to } n)+\#(\text { parts equal to } n-1) \geqslant n-1 \text {. }
$$

in the case of DPPs without special parts.

\subsection{The bijective construction}

Given some permutation matrix $A$ (i.e., some ASM without entries -1) of dimension $n$, set $k=1, A_{1}=A$, and repeat the following step $n-1$ times:

- Note down the number $a_{k}$ of rd-cells in the first row of $A_{k}$,

- delete the first row and the column containing the 1 (i.e., column $a_{k}+1$ ),

- rotate the matrix by $180^{\circ}$,

- let $A_{k+1}$ be the $(n-k) \times(n-k)$-matrix thus obtained, and increase $k$ by 1 .

(See Figure 5 for an illustration of this construction.)

Theorem 8. The sequence of $(n-1)$ numbers $\left(a_{1}, a_{2}, \ldots, a_{n-1}\right)$ obtained by the construction described above has the following properties:

- $0 \leqslant a_{i} \leqslant n-i$ for $i=1,2, \ldots, n-1$, i.e., $\left(a_{1}, a_{2}, \ldots, a_{n-1}\right)$ is an inversion word,

- $a_{1}=p(A)$,

- $a_{2}=q(A)-[q(A)+p(A) \geqslant n]$

- $a_{1}+a_{2}+\cdots+a_{n-1}=i(A)$.

Moreover, this construction gives a bijection between permutation matrices and inversion words.

Proof. It is clear that $0 \leqslant a_{k} \leqslant n-k$, since $a_{i}$ is (by construction) the number of rd-cells in the first row of an $(n-k+1) \times(n-k+1)$ permutation matrix, and this number cannot exceed $n-k$.

By construction, we also have $a_{1}=p(A)$.

Of the $q(A)$ lu-cells in the last row of $A$, precisely one will be deleted in the first step of our construction if and only if $q(A)+p(A) \geqslant n$ : By the rotation at the end of the first step, precisely these "surviving" lu-cells will turn up as the rd-cells in the first row of the matrix at the beginning of the second step, whose number will give $a_{2}$.

Finally, if there are $a_{k}$ rd-cells in the first row of the $(n+1-k) \times(n+1-k)$-matrix $A_{k}$ at the beginning of step $k$, then the column $a_{k}+1$ of $A_{k}$ contains precisely $a_{k}$ lu-cells, since the submatrix given by rows 2 to $n+1-k$ and columns 1 to $a_{k}$ must contain precisely $a_{k} 1$-cells. So in every step, the number of deleted $\mathrm{rd}-$ cells equals the number of deleted lu-cells, and after $n-1$ steps, 
- all of the rd-cells and lu-cells of $A$ (recall that their number is twice the number of rd-cells) have been deleted,

- and half of these cells have been noted down during the construction; their number is equal to $a_{1}+\cdots+a_{n-1}$.

It is easy to see that the mapping from permutation matrices to inversion words thus obtained is injective, whence it is a bijection.

Now we simply employ Striker's bijection [10] between inversions words and DPPs without special parts: This bijection maps an inversion word $\left(a_{1}, a_{2}, \ldots, a_{n-1}\right)$ to a DPP $\pi$ of dimension $n$ without special parts and with precisely $a_{i}$ parts $(n+1-i)$, whence we obtain

- $i(\pi)=a_{1}+a_{2}+\cdots+a_{n-1}$,

- $p(\pi)=a_{1}$,

- $q(\pi)=a_{2}+\left[a_{2}+a_{1} \geqslant n-1\right]$.

(the last assertion is due to the simple characterization (4)).

Theorem 9. The bijection $\Psi: \mathcal{A}_{n}^{0} \rightarrow \mathcal{D}_{n}^{0}$ established by the combination of Theorem 8 and Striker's bijection respects the triplet of statistics $(p, i, q)$.

Proof. The assertion is obvious for $i$ and $p$, so it remains to show this for $q$ : Letting $\epsilon:=\left[q(A)+a_{1} \geqslant n\right]$, we have $a_{2}=q(A)-\epsilon$ and thus obtain

$$
q(\pi)=q(A)-\epsilon+\left[(q(A)-\epsilon)+a_{1} \geqslant n-1\right] .
$$

We have to consider two cases:

- $\epsilon=1 \Longleftrightarrow q(A)+a_{1} \geqslant n$ : This implies $\left[q(A)-\epsilon+a_{1} \geqslant n-1\right]=\epsilon$.

- $\epsilon=0 \Longleftrightarrow q(A)+a_{1}<n$ : Observe that $q(A)+a_{1}=q(A)+p(A)=n-1$ is not possible for a permutation matrix $A$, since this would imply that the entries 1 in the first and last row appear in the same column. So we must have $q(A)+a_{1}<n-1$ in this case, whence we again obtain $\left[q(A)-\epsilon+a_{1} \geqslant n-1\right]=\epsilon$.

So in both cases, we have $q(\pi)=q(A)$.

\subsection{An open question}

Our construction was successful because we have the "translation" (4) of the (somewhat complicated) condition

DPP $\pi$ of dimension $n$ has a row of length $n-1$

for DPPs without special parts to an obvious (and quite simple) corresponding condition for matrices without entries -1 .

In the search for a "natural" bijection between ASMs and DPPs, it might be helpful to identify such "corresponding condition" for general matrices. 
Starting with the $7 \times 7$ permutation matrix corresponding to the permutation $\sigma=$ $(3,2,5,7,1,6,4)$, the pictures show the 6 steps of the bijective construction: The rows and columns to be deleted are indicated by thick gray lines. The inversion word thus obtained is $(4,2,1,1,0,1)$.

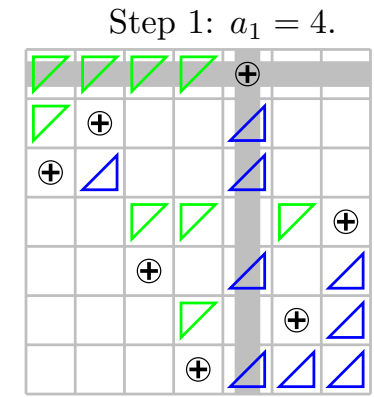

Step 4: $a_{4}=1$.

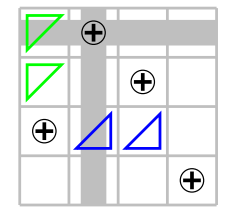

Step 2: $a_{2}=2$.
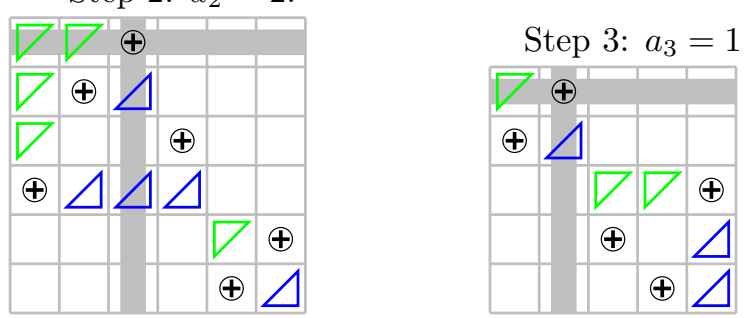

Step 5: $a_{5}=0$.

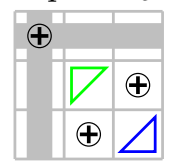

Step 6: $a_{6}=1$.

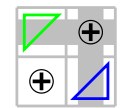

Figure 5: Illustration of the steps of the bijective construction.

\section{Acknowledgements}

I am grateful to the anonymous referee for many helpful comments.

\section{References}

[1] Arvind Ayyer. A natural bijection between permutations and a family of descending plane partitions. European Journal of Combinatorics, 31(7):1785 - 1791, 2010.

[2] Roger E. Behrend, Philippe Di Francesco, and Paul Zinn-Justin. On the weighted enumeration of alternating sign matrices and descending plane partitions. Journal of Combinatorial Theory, Series A, 119(2):331 - 363, 2012.

[3] Roger E. Behrend, Philippe Di Francesco, and Paul Zinn-Justin. A doubly-refined enumeration of alternating sign matrices and descending plane partitions. Journal of Combinatorial Theory, Series A, 120(2):409 - 432, 2013.

[4] David M. Bressoud. Proofs and Confirmations:The Story of the Alternating Sign Matrix Conjecture. Cambridge University Press, 1999.

[5] Greg Kuperberg. Another proof of the alternative-sign matrix conjecture. International Mathematics Research Notices, 1996(3):139-150, 1996.

[6] Greg Kuperberg. Symmetry Classes of Alternating-Sign Matrices under One Roof. Annals of Mathematics, 156(3):835-866, 2002. 
[7] Pierre Lalonde. Lattice paths and the antiautomorphism of the poset of descending plane partitions. Discrete Mathematics, 271(1-3):311 - 319, 2003.

[8] Pierre Lalonde. Alternating sign matrices with one -1 under vertical reflection. Journal of Combinatorial Theory, Series A, 113(6):980 - 994, 2006.

[9] W.H Mills, David P Robbins, and Howard Rumsey Jr. Alternating sign matrices and descending plane partitions. Journal of Combinatorial Theory, Series A, 34(3):340 - 359, 1983.

[10] Jessica Striker. A direct bijection between descending plane partitions with no special parts and permutation matrices. Discrete Mathematics, 311(21):2581 - 2585, 2011.

[11] D. Zeilberger. Proof of the alternating sign matrix conjecture. Electronic J. Comb., 3(\#R13), 1995. 